\title{
PLAN, DIVISIONS ET TABLE DES MATIĖRES.
}

\section{ExPOSTYON.}

Pages.

8 1. Sujet, but et division de cet ouvrage . . .

I.

\section{PRBMIÉRE PARTIB DB LOUVRAGE. INTRODUCTION.}

2. Sujet, but et division de cette introduction

CHAPITRE PREMIER.

ORIGINE ET TRANSFORMATION DES MYTHOLOGIES OU DES RELIGIONS NATURELLES.

\& 3. Origine des Religions et des Mythologies . . . . . . . . . . . . . 3

4. Transformations des Religions et des Mythologies . . . . . . . 4

8 5. Transformations de la Mythologie scandinave depuis son origine jusqu'à sa dissolution . . . . . . . . . . . . . . . . . 6

\& 6. Nature et caractères des Religions dans la période de l'Intuition . . 7

\& 7. Nature et caractères des Religions dans la période de la Raison . . 10

8. Nature et caractêres des Religions dans la période de I'Intelligence. If CHAPITRE II.

LA SCIENCE DE LA MYTHOLOGIE DANS L'ANTIQUiTÉ ET AU MUYEN AGE.

8ิ 9. Origine du système evhémériste. . . . . . . . . . . . . 16

8. 10. L'Evhémérisme chez les peuples de ia branche gète. . . . . . . 18

11. Systèmes mythico-historiques des savants au Moyen âge . . . . 19

8. 12. Odinn supposé d'origine thrâke et roi des Thrakes . . . . . . 21

8. 13. Odinn supposé d'origine turke et roi des Turks . . . . . . 23

14. Odinn réputé d'origine troyenne et roi des Troyens. . . . . . 27

CHAPITRE III.

SNORHI, SA VIE, ET L'HISTOIRE DE SES OUVRAGES.

8. 15. Vie, éducation et ouvrages de Snorri . . . . . . . . . . . . . . . 29

8. 16. Snorri est l'auteur de la Fascination de Gulfi . . . . . . . . . 31

8ิ 17. La Fascination de Gulfi composée avant le Heims-Kringla . . . . . 33

8. L'Intégrité du texte du Gylla-ginning . . . . . . . . . . . 35

19. Comment le Gylfa-ginning se trouve dans I'Edda en prose . . . . 38

CHAPITRE IV.

LE FOND hISTORICO-MYTHOLOGIQUE ET LA DISPOSITION DES NATÉHIALX DANS LE TRAITÉ DE SNORRI.

8. 20. Formation du sysième historico-mythologique de Snorri . . . 41

21. D'aprìs Snorri, les Ases sont originaires de I'Asie . . . . . . 43

22. Lage où les Ases ont vécu . . . . . . . . . . . . . . . . . . . 45

8. 23. Snorri expose les Mythes dans leur succession prétendue historique. 46

83 24. But plutot scientifique que littéraire du Traité de Snorri . . . . 49

CHAPITRE V.

FORMES LITTÉRAIRES, ENCADREMENT ET TITRE DU TRAITÉ DE SNORBI.

8. 25. La Poésie et la Science confondues ensemble . . . . . . . . . 50

26. Le Dialogue, la Discussion et les Joutes scientifiques . . . . . . . $\quad$. 52

27. Joute entre Vafthrodnir et Odinn; Guerre de la Wartbourg . . . 54 
* 28. La forme catéchétique de rEnseignement

39. Origine et raisons des Encadrements littéraires

30. Origine de l'Encadrement du Traité de Snorri.

3. 31. Titres d'ouvrages dans l'Antiquité, en Orient, et au Moyen âge . 71

3. 32. Titres douvrages norrains et du Traité mythologique de Snorri . 73

II.

DRUXIÉHE PARTIE DE L'OUVRAGR.

LA FASCINATION DE GULFI.

Traduction

III.

TROISIĖHE PARTIK DE L'OUVRAGE.

COMMENTAIRE CRITIQUE PERPÉTUEL.

$N^{\circ}$ (1) GULFI ET GẌFION.

*. 1. Gulfi, roi de la Marche-finne . . . . . . . . . 139

8. 2. Origine du nom de Séeland . . . . . . . . . . . . . $\quad$. 140

.8. 3ythe géologique et hiêratique sur le Séeland . . . . 1 110

2. 4. Bragi le Vieux et le style skaldique . . . . . . . . . . . . 142

8. 5. Le Mythe de Găfion fait partie intégrante de l'Encadrement . . . 144

No (2) GUI.FI VIKNT A ODINSEY.

\& 6. Voyage aérien de Gulf . . . . . . . . . . . . . . . . . . 144

\& 7. Forme extérieure de la Halle-des-0ccis . . . . . . 145

*. 8. La Halle-des-0ccis, d'après Snorri. . . . . . . . . . 147

$N^{\circ}$ (3) GULFI ENTRE DANS LA HAL,LE-DES-OCCIS.

z. 9. L'Intérieur de la Halle-des-Occis . . . . . . . . . . . 148

1. 10. Le Portier de la Halle-des-0ccis . . . . . . . . . . 149

8 11. Les Allées de la Halle-des-Occis . . . . . . . . . . . 149

3. 12. Les Portes ensorcelées de la Halle-des-0ccis . . . . . . 150

$\mathrm{N}^{\circ}$ (4) GOLFI DEVANT LES TnOIS CHEFS.

8. 13. Les Dieux Trinaires . . . . . . . . . . . . . 150

\& 14. Sublime, Équi-Sublime, et Troisième . . . . . . . 151

8े 15. Gulf en présence des trois Chefs . . . . . . . . . . 152

6. 16. Gulf provoque les trois Chefs . . . . . . . . . . . 153

N॰ (5) PĖRE UNIPERSEL ET SES DIPPÉRENTS NOMS.

8. 17. Les Divinités supremes . . . . . . . . . . . . . 154

8. Origine des noms propres et des noms épithétiques des Divinités 156

19. Explication des douze noms épithétiques d'Odinn . . . . . . 158

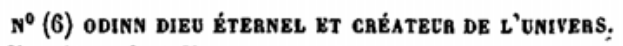

8. 20. Odinn dieu éternel et dieu conservateur . . . . . . . . . . 162

21. Odinn dieu créateur . . . . . . . . . . . . . . . . . . . . . 163

22. L'homme, l'œuvre principale du Créateur . . . . . . . . . 163

8. 23. Séjour de l'houme après sa mort . . . . . . . . . . 164

No (7) LE MONDE AVANT LE CRÉateug.

8. 24. L’origine de Père-Universel . . . . . . . . 165

8. 25. Les Thurses-Givreux et les Iotnes . . . . . .. 166

8 26. Ordre de formation des parties de la Mythologie 168 
$N^{\circ}(8)$ LA COSMOGONIE.

Pages.

3. 27. Le Bâillement-des-Hachoires . . . . . . . . . . . . 169

28. Le Séjour-Brumeux et les Vagues-Tempêtueuses . . 170

8. 29. Le Séjour de Gâte-Monde . . . . . . . . . . . 173

$\mathrm{N}^{\circ}$ (9) LE MONDE AVANT LES THURSES-GIVREUX.

8 30. La Matière morte est vivifiée. . . . . . . . . . . . . . . 174

8ิ 31. Nature, Garactère et Noms épithétiques d'Ymir . . . . : : 176

32. Époque glaciale du Monde primitif. . . . . . . . . . . . 176

$N^{\circ}$ (10) YMIR, PĖkE dES THURSES-GivBEUX.

8. 33. Ymir n'est pas un Dieu . . . . . . . . . . . . . . . . . . 177

34. La Génération spontanée, et l'immutabilité des espéces . . . . 178

8 35. Comment Ymir engendre les Parents des Thurses-Givreux . . . 180 No (11) LA TACHE AODHOMLA.

8 36. Le nom de la vache Audhumla . . . . . . . . . . $\quad$. . . 181

8े 37. Origine de la vache Audhumla . . . . . . . . . . . . . . . . 181

$\mathrm{N}^{\circ}$ (12) BÔMI ET SA RACE.

8ิ 38. Naissance de Bari . . . . . . . . . . . . . . . . . . . . . . 182

39. Bör le père d'Odinn . . . . . . . . . . . . . . . . . . . . . . 183

8 40. Les trois fils de Bor . . . . . . . . . . . . . . . . . . . . 185

No (13) LES Trols GÉNÉRATIONS DES THONSES-GiVREOX.

8 41. Les Représentants des trois générations primitives. . . . . . 186

42. Les Thurses-Givreux de la première race sont detruits . . . 187

N (14) CRÉATION DU MONDE ACTUEL.

8 43. Formation de la terre. . . . . . . . . . . . . . . . . . . . . 188

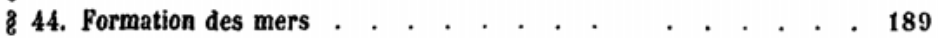

8 45. L'Arrangement du Ciel . . . . . . . . . . . . . . . . . 190

$N^{\circ}$ (15) ARBANGEMENT DE LA TERRE.

8 46. Le Séjour des Iotnes . . . . . . . . . . . . . . . . 191

8 47. L'Enclos-Mitoyen, et la Ferté Céleste . . . . . . . . . . 192

No (16) L'ANTHROPOGONIE MYTHIQUE.

8 48. L'Ethnogonie des Scythes . . . . . . . . . . . . . . . 193

8. 49. Arbres métamorphosés en hommes. . . . . . . . . . . 19.4

8. 50. Comment Snorri conçoit ce mythe anthropogonique . . . . . 196

8ิ 51. Hors-d'œuvre dans la réponse de Sublime . . . . . . . . . 196 $N^{\circ}(17)$ Nőv1 ET SES DESCENDANTS.

8. 52. Norvi et sa Glle Nott . . . . . . . . . . . . . . . . . . . 197

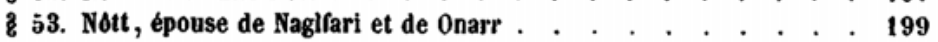

8. 54. Delling, troisième époux de Nott . . . . . . . . . . . . . . . . 200

$\mathrm{N}^{0}$ (18) MYTHES COSMOGoNo-ÉPIQUes SUR SOL et MANI.

85. Mundilfari, père de Sol et de Mani . . . . . . . . . . . . 201

8. 56. Sol, la fiancée de Glèn; ses deux chevaux . . . . . . . . . 203

8. 57. Les phases et les taches de la lune . . . . . . . . . . . 203

8. 58. Soleil et Lune, dans l'origine divinités zoomorphes . . . . . 204

$$
\mathbf{N}^{0} \text { (19) LA FUITE dE SÓL ET DE MANI. }
$$

8 59. Un mythe de la période primitive 
Pages.

60. Les lotnes zoomorphes . . . . . . . . . . . . . . 20s

8 61. Les loups iotuiques Skoll et Hati . . . . . . . . . . . . . . . . 209

$\mathrm{N}^{\circ}$ (21) LES GÉANTES MĖnES ET NOURBICIĖRES DES LOUPS.

8. 62. Les Géantes zoomorphes. . . . . . . . . . . . . . . . . . . . 209

83. Le Bois de Fer, et Mana-Garmr. . . . . . . . . . . . . . 210

No (22) LA NATORE DE L'ARC-EN-CIEL.

84. La Voie-Tremblotante, et les Bains de Bassin . . . . . . . 211

\& 65. Comment Snorri conçoil Bifrőst . . . . . . . . . . . 212

$\mathrm{N}^{\circ}$ (23) PREMIER AGE DES A8ES; LES DVERgS.

8 66. Jeunesse des Ases. . . . . . . . . . . . . . . . . . . 213

8. 67. Le prétendu Age dor des Ases. . . . . . . . . . . . 214

8 68. Les Kvarkes des Scythes et des Gètes . . . . . . . . . 217

8 69. Les Dvergs dans la Mythologie norraine . . . . . . . . . 218

8 70. Origine et races des Dvergs, d'après Snorri . . . . . . . . . 222

No (24) LE FRÊNE d'yggdBASILL; LA FONTAINE dE MIMIR.

8 7I. Le Frêne d'Yggdrasill, Arbre d'Établissement et de Jugement . . 223

8. Le Frene d'Yggdrasill, Arbre de Vie . . . . . . . . . . 225

8 73. Le Frêne d'Yggdrasill, d'après Snorri. . . . . . . . . . 227

8 74. La Fontaine de Mimir. . . . . . . . . . . . . . . 228

$N^{\circ}(25)$ LES NOBNES; LA YONTAINE D'URDUR.

8 ?. La Prédiction du Destin . . . . . . . . . . . . . . 230

8. 76. Les Nornes, Arbitres de la Destinée . . . . . . . . . . 232

8 77. Les Nornes épiques . . . . . . . . . . . . . . . . . . 233

$N^{\circ}(26)$ L.KS MERVEILI.ES ET LES SODFFRANCES DU PBÊNE D'YGGDASILL..

8. 78. L'Aigle, et le Dragon Nidhogg . . . . . . . . . . . . . . . . . 234

8 79. L'Écureuil Ratakostr, et le Cerf broutant . . . . . . . . 235

8 80. La Tonbée de miel, et les Cygnes de la Fontaine d'Urdur . . . 237

$\mathrm{N}^{\circ}$ (27) LES DEMEUUES cÉLESTES.

8 81. Le Sẹjour des Alles; Large-Éclat; Ĺtincelant . . . . . . . . 238

88 82. Roches-Célestes; Chaumine de Vali; Chaumine aux l'ortes; et Brillant . . . . . . . . . . . . . . . . . . 240

$\mathrm{N}^{0}$ (28) ORIGINE DES VENTS BT DES SAISONS.

8 83. L'Aigle Hræsvelg . . . . . . . . . . . . . . . . . . 242

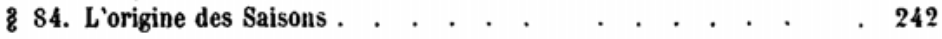

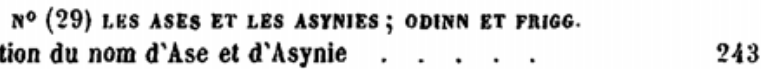

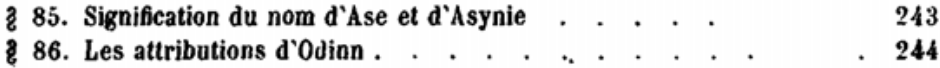

87. Les noms épithètiques d'Odinn. . . . . . . . . . . . . . . 246

8 88. Conception et attributions de Frigg $\quad$. . . . . . . . . . . . . . $\quad 250$

No (30) CONCEPTION DU DIEV THOR, ET SES ATrRibUtIONS.

8 89. Pirkunis, Firgunis, liörgynn . . . . . . . . . . . . . . . . . 251

8 90. Les noms de Thor, de Thor au Char, de Thor aux Ases . . . . 253

8 91. La Demeure de Thor . . . . . . . . . . . . . . . . 254

8 92. Les Boucs de Thor

93. Le Martean de Thor; ses Gantelets de fer, et sa Ceinture de Force . 256 
$N^{\circ}$ (31) BAL.DUR; NIÖ́DUR; SKADd; FRETR; ET PHEYIA.

Pages.

ริ 94. Baldur, dédoublement et héritier du dieu Soleil . . . . 258

8. 95. Niőrdur, dédoublement du Dieu du Soleil . . . . . . . 260

8̊ 96. Skadi, héritière de la Déesse Vrindus. . . . . . . . . . . 262

8ุ 97. Freyr, dédoublement et héritier du Dieu du Soleil . . . . . . 265

8. 98. Freyia, dédonblement et héritière de la Déesse de la lıune . . . 266 $N^{\circ}$ (32) TYR; BRAGI; ET IDUNN.

3. 99. Tyr, originairement le dieu Ciel . . . . . . . . . . . . . 268

8ิ 100. Bragi, dédoublement et héritière de la Lune . . . $\quad$. . 272

8ै 101. Idunn, l'Épouse de Bragi . . . . . . . . . . . . . . 273

$\mathrm{N}^{\circ}$ (33) HEIMDALL; HÖDUR; VIDAR; ULLR; FORSETI.

\& 10 . Origine stellaire de Heimdall . . . . . . . . . . . . . 274

I (3. Origine solaire de Vidarr . . . . . . . . . . . . . . 277

104. Hodur, ancien Dieu du Combat . . . . . . . . . . . . 280

1 (5. Origine solaire du Dieu-héros Ullr . . . . . . . . . . . . . 282

106. Vali, ancien Dieu de la Mort . . . . . . . . . . . . 283

8. 107. Forseti, dédoublement de Baldur . . . . . . . . . . 284

$\mathrm{N}^{0}$ (34) LOKI, HEL ET LE SERPENT DE L'ENCLOS-MITOYEN.

\& 10 . Loki, Symbole de l'Astre du Soir . . . . . . . . . . . . 284

8. 109. Le Serpent de l'Enclos-Mitoyen, Symbole de l'Océan ... . 286

\& 110 . Hel, originairement Déesse de la Lune . . . . . . . . . . 287

8 111. Signification symbolique du Loup de Penrir . . . . . . . . . 298

\& 11?. Les Ases enchatnent lc Loup de Fenrir . . . . . . . . . . 288

$N^{\circ}$ (36) LES ASYNIES.

8 113. L'Asynie Saga, spécialisation de la Norne Urdur . . . . . . . 290

8 11\%. Cure, dédoublement de Freyia . . . . . . . . . . . 291

8ิ 11. Gäfion, dédoublement de Freyia . . . . . . . . . . . 291

8ิ 113. Fulla, dédoublement de Frigg . . . . . . . . . . . . . . . 292

8 11. Freyia, héritière de Rindur et de Frigg . . . . . . . . . . 293

$\mathbf{N}^{0}$ (37) SIÖFN ; LOFN ; VÖR; SYN ; HLIN ; SNOTRA ; GNÂ.

8 118. Siofn, dédoublement de la Déesse Thiuth . . . . . . . . . . 294

11). Lofn, dédoublement de Preyia . . . . . . . . . . . . 295

8ิ 121. Vör, dédoublement de la Déesse Taviti . . . . . . . . 295

\& 12. Syn, dédoublement de la Déesse Taviti. . . . . . . . . 296

8ิ 12:. Hlin, dédoublement de Frigg . . . . . . . . . . . . . . 297

8ิ 123. Snotra, spécialisation de Freyia . . . . . . . . . . 297

\& 12. Gnâ, dédoublement de Freyia . . . . . . . . . . . . 297

No (38) LES VALKYRIES; LES ASYNIES IÖRD ET NINDUR.

8. 12i. Les Valkyries conçues d'après les Alhi-runes historiques . . . 298

8 12i. Les Asynies Iőrd et Rindur . . . . . . . . . . . . . 300

$N^{\circ}$ (39) HYMIR; LES FIANÇAILLES DE FREYR ET DE GERDUR.

8 12. Gymir, Örboda et Gerdur . . . . . . . . . . . . . . . . . . 302

8 121. Les fiançailles de Freyr et de Gerdur . . . . . . . . . . 304

\& 12!. Les éléments épiques ajoutés an niythe symbolique . . . . . 305 


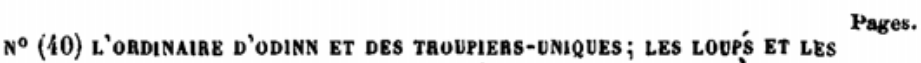
COBBEAUX D'ODINN.

8. 130. Sæhrimnir, Andhrimnir et Eldhrimnir . . . . . . . . . 306

8 131. Odinn boit du vin . . . . . . . . . . . . . . . . . . . 308

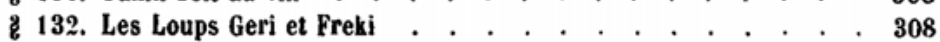

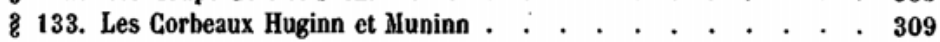

No (41) LA CHĖVRE HEIDRUNE, L'ARBRE LERAD, ET LE CERP EIKTHYRNIR.

8ి 134. La Chèvre Heidrane . . . . . . . . . . . . . . . . . . . 309

8 135. LArbre merveilleux Lærad . . . . . . . . . . . . 310

8 136. Le Cerf Eikthyrnir . . . . . . . . . . . . . . . . . . 311

$N^{\circ}(12)$ LA HALLE-DES-OCCIS; LES TBOUPIERS-UNIQUES; TYPES DES CHOSES, AU CIEL.

8 137. Grandeur de la Halle-des-0ccis . . . . . . . . . . , 312

8े 138. Jeux des Troupiers-Uniques . . . . . . . . . . . . . . 312

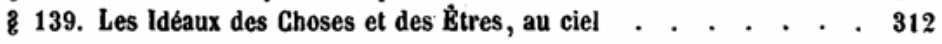

No (43) L'IOTNE CONSTROCTEUR; SVADILFARI; SLEIPNIR; SKID-BLADNIR.

8 140. Le Géant-des-Montagnes, architecte. . . . . . . . . . 313

8े 141. Svadilfari et Sleipnir . . . . . . . . . . . . . . . . 314

8 142. Le navire Sktdbladnir . . . . . . . . . . . . . . . . . 314 $N^{\circ}$ (44) AVENTURES dE THÓ DANS LE SÉJOLA DES IOTNES ET DANS L'ENCL.OSEXTÉraieur.

8 143. Thor s’adjoint Thialfi et Röskva . . . . . . . . . . . . . . 315

8 144. Thor et Skrymir . . . . . . . . . . . . . . . . . . 317

8. 145. Thor chez Loki de l'Enclos Extérieur . . . . . . . . . . 319 $N^{\circ}(45)$ THÓ PÉCHE AVEC hYMIB, ET COMBAT LE SERPENT-DE-MER.

8 146. Thor et le Géant Hymir . . . . . . . . . . . . . . . 326

8 147. Lutte de Thor contre le Serpent-de-Mer . . . . . . . . . . 326 Nod46) PLESSENTIMENTS, MORT, ET FUNÉkAILLES dE BAL.dUR.

8 148. Mort de Baldur . . . . . . . . . . . . . . . . . . . . . . 327

\& 149. Funérailles de Baldur . . . . . . . . . . . . . . . . . . . . 329

8 150. Baldur ne peut revenir de Hel . . . . . . . . . . . . . 331

$\mathrm{N}^{\circ}$ (47) PUITE, PAISE ET PONITION DE LOKI.

8 151. Loki pris par les Ases . . . . . . . . . . . . . . . . . . 332

8े 152. Punition de Loki . . . . . . . . . . . . . . . . . 334

N० (48) LE CRÉPUSCULE DES GRANDEURS.

8 153. Le terrible Hiver . . . . . . . . . . . . . . . . . . 335

8 1 1 4. Les scènes successives du Drame terrible . . . . . . . . . . 337 No (19) LA hENAISSANCE DU CIEL, DE LA TEHRE, DES DIEUX, ET DES hOMMES.

8 155. Renaissance de la Terre . . . . . . . . . . . . . . 339

\& 156. Restauration du Ciel . . . . . . . . . . . . . . 340

8 157. La nouvelle race humaine; le nouveau Soleil . . . . . . . 341

$N^{\circ}$ (50) ÉPILOGDE DE SUQLIME. REPRISE ET FIN DE L'HISTUIRE DE L'ENCADREMENT.

8 158. L'Épilogue de Sublime . . . . . . . . . . . . . . . . . . . . 342

8 159. Gult enseigne en Suède ce qưil a appris de Sublime . . . . 342 